\title{
Estrategias didácticas para revitalizar las lenguas originarias en estudiantes de la Universidad Nacional Intercultural de la Amazonía.
}

\section{Didactic strategies to revitalize native languages in students of the National Intercultural University of the Amazon}

\begin{abstract}
Autores
Abdías Chávez Epiquén. Universidad Nacional Intercultural de la Amazonía. Perú. innovasciencia@gmail.com http://orcid.org/0000-0001-5589-5217
\end{abstract}

Manuel Chenet Zuta. Universidad Nacional Altoandina de Tarma.Perú.innovaarticle@gmail.com https://orcid.org/0000-0003-2088-2541

Walter Quispe Cutipa. Universidad Nacional Intercultural de la Amazonia. Perú. article.innova@gmail.com http://orcid.org/0000-0001-9355-1984

Fecha de recibido: 2019-08-06

Fecha de aceptado para publicación: 2019-09-16

Fecha de publicación: 2019-09-30

\begin{abstract}
Resumen
El uso de lenguas originarias ha disminuido en las etnias de la región Ucayali, los jóvenes rehúsan hablar en su lengua, por ello el presente estudio tiene el objetivo de fomentar la revitalización de las lenguas originarias en estudiantes del segundo ciclo de la carrera de Educación Inicial Bilingüe de la Facultad de Educación de la Universidad Nacional Intercultural de la Amazonia a través de la aplicación de estrategias didácticas. La investigación fue descriptiva, explicativa, con diseño preexperimental de enfoque mixto; y una muestra de 22 estudiantes. En la prueba de entrada, 22 estudiantes se hallaron en el nivel de proceso y la media 9.73. La prueba de salida, el 69\% alcanzó logro previsto, la media 16.6; se probó la hipótesis a nivel de 0,05 de significancia. Cada estudiante tradujo un tema a una lengua originaria, aprendió la entonación; primero versos, luego estrofas. Se concluyó que las experiencias interculturales fueron exitosas en la revitalización de lenguas en los estudiantes de Educación Inicial Bilingüe.
\end{abstract}

Palabras clave: Experiencias interculturales; revitalización lingüística; lengua originaria.

\begin{abstract}
The use of native languages has decreased in the ethnic groups of the Ucayali region, young people refuse to speak in their language, so this study aims to promote the revitalization of native languages in students in the second cycle of Bilingual Initial Education of the Faculty of Education of the National Intercultural University of Amazonia through the implementation of teaching strategies. The research was descriptive, explanatory, with pre-experimental design of mixed approach; and a sample of 22 students. In the entrance test, 22 students were at the process level and the average was 9.73 . The exit test, $69 \%$ reached expected achievement, mean 16.6; the hypothesis was tested at level 0, 05 of significance. Each student translated a subject into a native language, learned intonation; first verses, then stanzas. It was concluded that the intercultural experiences were successful in revitalizing languages in the Bilingual Early Education students.
\end{abstract}

Key words: Intercultural experiences; linguistic revitalization; native language. 


\section{Introducción}

La cultura como conjunto de costumbres, estilos de vida y tradiciones diversas de los pueblos, es un tema transversal de la educación peruana. Las aulas de clase están conformadas de personas con diferentes culturas, valores y costumbres que marcan una identidad. En la tarea de educar, los profesores representan la interculturalidad solamente con la exhibición de un traje ocasional o una música típica $y$, piensan que se fomenta la interculturalidad. Lo cierto es que, abordar el estudio de una cultura remonta a situaciones mucho más profundas de la vida y de la cosmovisión del hombre.

Las diferentes culturas tienen que ver con una característica del ser humano: animales sociales. El hombre no puede vivir solo y desde tiempos pasados, una de las claves de la supervivencia consistió en organizarse en grupo, dominar los retos que impone la naturaleza a través de la inteligencia y de combinar fuerzas para buscar soluciones. "Gracias al patrimonio que conserva cada pueblo, cada ciudad ... se puede llegar a conocer y dado que no podremos hablar con todos los individuos, observaremos lo que tienen, lo que conservan y cuidan.," (Santacana, 2014, p. 49).

En tal sentido, la sociedad se caracteriza por el reconocimiento de los derechos fundamentales como ser humano, aspectos que no se puede quitar o negar de manera lícita. Es el Estado quien debe garantizar y respetar estos derechos, sin depender de la cultura que proviene, ni de la nacionalidad de la persona. Según el artículo $1^{\circ}$ de la Declaración Universal de Derechos Humanos (1948) estos mismos derechos son garantizados en el siguiente tenor: "todos los seres humanos nacen libres e iguales en dignidad y derechos y, dotados como están de razón y conciencia, deben comportarse fraternalmente los unos con los otros" (p.1).

En el Perú, según el MINEDU (2017) existen 47 lenguas originarias que son habladas por cuatro millones de personas, de hecho, es el país con más diversidad lingüística y cultural, reúne aproximadamente 55 etnias indígenas u originarias. Según su extensión o alcance las lenguas nativas se clasifican:

- Lenguas de uso regional o departamental. Habladas generalmente en el ámbito regional o departamental.

- $\quad$ Lenguas de uso provincial. Los hablantes son en su mayoría naturales de la provincia.

- $\quad$ Lenguas de habla distrital. Habladas en territorio de en un distrito.

Se encuentran lenguas nativas $u$ originarias que se convierten en un símbolo de identidad de una región, provincia o distrito. El aimara por ejemplo es una lengua hablada en la región Puno, es una lengua regional; en Apurímac el quechua. En la provincia de Condorcanqui (Amazonas) se habla el awajún, es una lengua provincial. En el caso de la lengua ashaninka es representativa al distrito de Coviriali de la provincia de Satipo, lengua distrital. En el Perú, el quechua es la lengua con mayores hablantes, pues se habla en varias regiones del país. También el ashaninka, se ha identificado que se habla en 9 regiones; el awajún en 7 regiones; el shipobo en 5 y; el matsigenka solo en 4 regiones (MINEDU, 2017). 
El MINEDU (2017, p.52) manifiesta que: "Las 47 lenguas originarias pueden ser agrupadas en lenguas vitales, lenguas en peligro, y lenguas seriamente en peligro". Estas clasificaciones han tenido como base la nomenclatura de la UNESCO, además de consultar a los representantes de los pueblos y a los investigadores (lingüistas) para tener en cuenta las modificaciones:

- Lenguas vitales. Las que son habladas de generación en generación en la misma comunidad lingüística, donde su transmisión es ininterrumpida de una generación a otra. En este nivel, se considera a las lenguas que son habladas en casi todos los ámbitos de la comunicación.

- Lenguas en peligro. Son las que se habla en la mayoría de las personas adultas de la comunidad lingüística. En este caso, la lengua se restringe a ciertos espacios de la comunicación y la forma de transmitir solo es parcial en ciertas comunidades.

- Lenguas seriamente en peligro. Son las que se hablan, solo por las personas adultas mayores, o sea de la tercera edad y de modo parcial, con escasa frecuencia y de manera restringida para otros ámbitos comunicativos. Estas, ya no son transmiten a las generaciones.

De igual manera, Stanton, Hall, Carjuzaa (2018, p. 11) exponen que: "los esfuerzos de revitalización dentro de las escuelas son difíciles, ya que las perspectivas eurocéntricas siguen siendo dominantes, especialmente en la educación en estudios sociales, y los mitos y conceptos erróneos se perpetúan fácilmente". Este contexto pone de manifiesto el alto riesgo de la desaparición de diversas lenguas, como indica la (UNESCO, 2003):

Una lengua está en peligro cuando sus hablantes dejan de utilizarla, cuando la usan en un número cada vez más reducido de ámbitos de comunicación y cuando dejan de transmitirla de una generación a la siguiente. Es decir, cuando no hay nuevos hablantes, ni adultos, ni niños (p.2).

Por lo que se debe enfatizar sobre la importancia de las lenguas originarias, las cuales son preservadas a través de la memoria, para los pueblos indígenas es muy importante conservar sus dialectos pues son fuentes de cultura, tradición e historia. El legado lingüístico, cultural y cognitivo principalmente se transfiere a los jóvenes y niños mediante la oralidad, "la pérdida de la memoria equivale a la pérdida de la historia personal; provoca el naufragio de los pensamientos, tradiciones y saberes ancestrales" (Jiménez, 2017. p 259). Si no se mantiene el uso de una lengua, o se practica, ni se enseña desde las escuelas o por las familias, esa lengua estará en peligro de extinción, significa que tarde o temprano esa cultura puede extinguirse (Herrán y Rodríguez, 2017).

El hecho real, es que existen pueblos indígenas que se han resignado a la desaparición de su lengua, por consiguiente, saben que su cultura irá desvaneciéndose con ella, no obstante, existen personas miembros de las comunidades indígenas que tratan de frenar esta situación, al respecto Fitzgerald (2017, p. 292) explica que: "la revitalización y la recuperación de las lenguas actúan como un factor de protección y transmiten resiliencia a las comunidades en las que existen disparidades significativas".

De igual manera, es necesario reconocer el papel protagónico que tiene la escuela en esta situación, ya que debe ser garante de la inclusión educativa. Como expresa Uriarte (2006, p.8) "La escuela falla cuando no da respuestas adecuadas a las necesidades educativas de todos los alumnos y no compensa las limitaciones de origen familiar o social que afecta a alumnos 
procedentes de familias problemáticas y de medios desfavorecidos". Tomando en consideración esta perspectiva (Ávila, 2018) expresa:

La cultura se concibe como una forma de vida característica de una comunidad, en la cual se incluye un conjunto de prácticas relativamente estables y poco cambiantes que se heredan de padres a hijos. Los conocimientos y formas de hacer que los estudiantes desarrollan cuando participan en esas prácticas, por lo tanto, constituyen su herencia cultural (p. 179).

Es preocupante la realidad en materia académica en la Universidad Nacional Intercultural de la Amazonia, su creación obedece a la necesidad de atender directamente a las personas de las comunidades nativas y brindarles la oportunidad de ser profesionales sin perder su lengua materna. En el artículo 1 de su estatuto, la UNIA (2016) se define como: "Institución Educativa Superior de carácter intercultural constituida por docentes y estudiantes al servicio de la Amazonia y el país" (p.4). Asimismo, en la Ley $\mathrm{N}^{\circ} 27250$ (1999), Ley de creación de la Universidad Nacional Intercultural de la Amazonia, en su artículo $\mathrm{N}^{\circ} 2$, de los fines de la Universidad indica que: a) Prestar atención profesional completa, investigación científica y acciones culturales en toda su extensión de los pueblos etnolingüísticas de la amazonia; b) Generar desarrollo de modo sostenible, conservar el potencial pluricultural de la amazonia; c) Mayor contribución social, interpretación, preservación y difusión de las culturas indígenas en un ámbito de diversidad y pluralidad amazónica cultural (p.1).

Para el ingreso a la Universidad Nacional Intercultural de la Amazonia se determina un porcentaje de $80 \%$ de postulantes de lengua originaria y $20 \%$ de cultura mestiza o hispanohablantes, lo que la convierte en una universidad multi lingüística. Las culturas con mayor representatividad en la universidad son; shipibo, el awajun y ashaninka. En menor proporción se tiene estudiantes gines, wampis, kokama, achuar, quechua, shawi, matsigenka. Debido a este contexto multicultural se presentan dificultades para lograr competencias apropiadas en la experiencia educativa, puesto que una gran mayoría de los docentes, sobre todo en la Facultad de Educación de la UNIA, no son bilingües, son monolingües.

Al analizar la procedencia de los estudiantes de la UNIA se observa que la transculturización del español y la modernidad vigente ha disminuido la continuidad del uso de las lenguas originaras, hay pueblos indígenas donde solo las personas mayores hablan su lengua, así mismo, existen comunidades donde no hay niños que hablan su lengua indígena, y también existen pueblos indígenas donde hay un buen número de hablantes, pero se encuentran dispersos en diferentes comunidades.

En este contexto, nace el propósito de ensayar diversas estrategias para mantener vigente el uso de la lengua originaria, paulatinamente el Ministerio de Educación ha puesto en marcha muchos programas para la educación básica regular, así como también ha publicado textos educativos en lengua originaria a fin de promover el uso de las lenguas originarias. Sin embargo, aún no existe la verdadera motivación para que el poblador indígena se sienta orgulloso de su cultura. En la UNIA docentes y estudiantes han puesto en práctica proyectos para el tratamiento de las lenguas indígenas, en asignaturas destinadas a desarrollar habilidades lingüísticas para la redacción y expresión, pero los resultados son muy pobres.

El presente estudio tiene como objetivo: fomentar la revitalización de las lenguas originarias en estudiantes del segundo ciclo de la carrera de Educación Inicial Bilingüe de la Facultad de Educación de la Universidad Nacional Intercultural de la Amazonia a través de la aplicación de 
estrategias didácticas, con actividades en el aula que implican el uso de la música, el canto y la traducción.

\section{Metodología}

De acuerdo a su finalidad se trata de una investigación cuantitativa, de tipo aplicada, nivel descriptivo explicativo y diseño pre experimental. La unidad de análisis del estudio estuvo constituida por estudiantes del segundo ciclo de la carrera de Educación Inicial Bilingüe de la Facultad de Educación de la Universidad Nacional Intercultural de la Amazonia en dos periodos académicos. El tamaño de muestra fue determinado a criterio del investigador siendo de 22 estudiantes que cursaban el segundo ciclo. Tomando lo mencionado por Hernández et al. (2006) la técnica de muestreo utilizada fue no probabilística, siendo deliberada o intencional.

Sabiendo que los estudiantes del segundo ciclo de la carrera de Educación Inicial Bilingüe de la Universidad Nacional Intercultural de la Amazonia, cursan la asignatura: Tratamiento de Lengua Indígena I, y la sumilla exige como competencia la concientización y fortalecimiento de la lengua originaria, teniendo como el tema eje la revitalización de la lengua indígena; así se proporciona un contexto ideal para la aplicación del estudio, por ende, en el lapso de todo el semestre en las sesiones de aprendizaje se emplearon actividades académicas enfocadas al desarrollo de la competencia de revitalización lingüística.

Puesto que la mayoría de los estudiantes presentan edades comprendidas entre 18 y 21 años, las características de estos jóvenes, absorbidos por la modernidad, es el uso frecuente de las redes de información, de celulares y aplicativos electrónicos de comunicación masiva. Por lo cual, resulta complicado sugerirle que se identifique con su cultura y que se exprese con fluidez en su lengua originaria, ya que en cierto modo le avergüenza hacerlo. Por tanto, se optó por aplicar estrategias didácticas que consistieron en:

- Elegir una canción favorita, la que tiende a escuchar con mayor frecuencia.

- Transcribir y aprender de memoria el contenido estrofa por estrofa.

- Traducir a una lengua materna, la más conocida para el estudiante

- Articulizar, la pronunciación.

- Oralizar la canción, cantar en la lengua materna y en español.

Las estrategias se enfocan en que cada alumno exteriorice sus gustos en la moda, la música, la comida, entre otros, a fin de motivarlos a realizar las actividades de interculturalidad, e ir introduciendo dentro de su perfil el sentido de pertenencia por sus lenguas originarias. Este momento no fue para identificar a que cultura étnica pertenece, sino para conocer sus preferencias y a partir de ello encaminar hacia la revitalización de las lenguas originarias.

Para realizar la aplicación de las estrategias didácticas en el aula, se aplicó una evaluación mediante una prueba de entrada y salida, es decir un pre test antes de la aplicación y otra medición después de las estrategias. Para la descripción de los resultados se utilizaron tablas de contingencia y figuras de barras, para la prueba de hipótesis se recurrió a la prueba de $\mathrm{t}$ de Student por diferencia de medias para muestras relacionadas. 


\section{Resultados y discusión}

El promedio en la prueba de entrada Pre test fue de 9.73, con una desviación de 14.14. Mientras que, en la prueba de salida, el promedio es de 16.6 con una desviación de 1.76 sobre la revitalización de lenguas, consultar Tabla 1 (en Anexos).

En los niveles de logro, en el caso del Pre test, el 45\% se ubica en el nivel de inicio y el $55 \%$ se encuentra en nivel de proceso. Para el Pos test, el 14\% se encontró en el nivel de proceso, el $59 \%$ en el nivel de logro previsto y el $27 \%$ alcanzó un nivel de logro destacado. Esto en cuanto a la competencia de revitalización de lenguas indígenas en la UNIA. La diferencia es bastante significativa.

La mayor parte de estudiantes en el pre test, no tomaron interés en poner en práctica sus conocimientos de lengua materna para usarlo en hechos cotidianos, como ensayar una canción conocida, entre otros temas dialógicos. En la prueba de salida se observa que un gran porcentaje ha tomado conciencia de su cultura, se interesa por ejercitar su lengua materna, se le ha despertado el interés de difundirla, y de publicar su cultura. Consultar Tabla 2 (en Anexos).

Con referencia a probar la hipótesis, se utilizó de la prueba T de Student para muestras relacionadas de diferencia de medias, ya que la variable posee una escala de intervalos que los puntajes de la muestra poseen una distribución normal.

La prueba $\mathrm{T}$ de Student arroja un valor de $00>0,05$, por ello, aceptamos la hipótesis alterna $\mathrm{H}_{1}$ y se rechaza la hipótesis nula $\mathrm{H}_{0}$, la conclusión es que, sí existe diferencia significativa de la prueba de entrada y de salida, que las experiencias didácticas interculturales han influido exitosamente en la revitalización de las lenguas indígenas de los estudiantes del segundo ciclo de Educación Inicial Bilingüe de la Universidad Nacional Intercultural de la Amazonia.

En el Pos test, el 14\% se encontró en el nivel de proceso, el 59\% alcanzó un nivel de logro previsto, mientras que el $27 \%$ alcanza un nivel destacado. El promedio de la prueba de entrada fue de 9.73, y en la prueba de salida se obtuvo un promedio de 16.6, significativo al 0,05 es decir un 5\% a favor del investigador. El nivel de significancia menor a lo previsto, al nivel de error a aceptar, $(0,00)$, es un P-valor menor que 0,05 por lo que se concluye que existe diferencias.

Las experiencias didácticas interculturales ayudan favorablemente en la revitalización de lenguas indígenas en estudiantes del segundo ciclo de Educación Inicial de la Universidad nacional Intercultural de la Amazonia; al 0,05 de significancia y p-valor $=0$, es decir 5\%de error y $95 \%$ a favor de la investigación.

El 14\% está en proceso de adoptar la revitalización de una lengua indígena; por que la identificación de un tema de interés basado en acciones interculturales ayuda favorablemente en la revitalización de una lengua indígena.

El 59\% de los estudiantes del segundo ciclo de Educación Inicial Bilingüe ha logrado el nivel previsto en la traducción de una lengua indígena; es decir, la traducción textual de una canción de interés del estudiante ayuda favorablemente en la revitalización de lenguas originarias.

El 27\% de los estudiantes del segundo ciclo de Educación Inicial Bilingüe se ha destacado en la traducción y entonación de una canción de su interés; por lo que se convierte como estrategia didáctica para la revitalización de lenguas indígenas.

El método de identificación de temas de interés del estudiante, la traducción y entonación de canciones de su preferencia a una lengua originaria, así como el dialogo entre pares y los 
intercambios de información cultural en el aula, ayudaron a fortalecer las actitudes de los alumnos para tomar conciencia de su identidad. Que se demuestra: "en el afianzamiento de la identidad cultural, el orgullo étnico y la activación de una enseñanza, que desde los centros docentes la mantenga viva y en equilibrio dinámico entre los dos polos culturales: el indígena originario y el contexto hispanohablante" (Herrán y Rodríguez, 2017 p. 164).

De igual manera, el docente se puede afianzar en lo propuesto en la Constitución del Perú y la Ley de Lenguas (Ley $\mathrm{N}^{\circ}$ 29735) donde se manifiesta que todas las lenguas indígenas son oficiales en las zonas donde predominan. Así mismo, el Estado está obligado atender e implementar propuestas sobre la revitalización de las lenguas indígenas en los organismos públicos instalados en las zonas bilingües del país. Esta propuesta es ratificada por Becerra, Mayo \& Fones $(2019$, p.15) cuando manifiestan que: “...las políticas públicas deben fortalecer el papel y el prestigio de los educadores de lenguas indígenas y desestimar de manera equitativa la labor de revitalización de las lenguas entre todos los participantes".

En Latinoamérica, se introdujeron en los programas educativos el reconocimiento de la diversidad sociocultural $\mathrm{y}$, se han implementado planes educativos encaminados hacia la revitalización de las lenguas originarias, en el marco de este contexto se reconoce los derechos educativos y culturales de los pueblos originarios (Turra y Ferrada, 2016).

Como se observa, el análisis conlleva a un problema social, como es la perdida de ciertas lenguas, con el trascurrir del tiempo este fenómeno es considerado de alto riesgo para la humanidad, ya que este es un derecho constitucional, proteger a las diferentes culturas vivas de una sociedad. Autores como Embriz y Zamora (2012) reconocen que la desaparición de una lengua es una perdida irremediable para la humanidad, la variedad lingüística representa un patrimonio para la humanidad al encerrar la esencia cultural de un pueblo, con esta desaparece la cosmovisión de un pueblo; se pierden tradiciones, identidad, rituales, y diversos aspectos culturales presentes en la oralidad.

Por otra parte, las políticas públicas adoptadas por el sistema educativo, juegan un papel importante en relación al reconocimiento de la diversidad, la heterogeneidad de las familias y de las comunidades que participarían en estos procesos, ya que no todas las comunidades y familias apoyan el renacimiento de las lenguas indígenas, unas se resisten a hacerlo en el contexto escolar, y otras no cuentan con los conocimientos ancestrales para colaborar como se requiere. (Becerra, Mayo \& Fones (2019).

La función docente ocupa una responsabilidad didáctica para motivar, estimular y proponer la reproducción de la lengua originaria mediante temas cotidianos, cánticos, discursos entre otras actividades importantes para el educando. Ramírez y Suárez (2016) explican que para potenciar la enseñanza de las lenguas originarias: "se necesita la formación de profesores totalmente capacitados lingüísticamente y pedagógicamente y que promuevan no solo la enseñanza del idioma, sino el respeto a la cultura" (p. 385).

Al respecto, Kroskrity (2018, p.11) en su estudio concluye que: "La resistencia de los ensamblajes lingüísticos e ideológicos indígenas que he descrito aquí sugiere firmemente que tales patrones proporcionan modelos indígenas importantes para proyectos de revitalización de las lenguas dirigidas a las lenguas en peligro de extinción”. A lo que Hermes, Bang y Marín (2012, p.398) incluyen lo siguiente:

El caso de la revitalización de las lenguas indígenas es un microcosmos de la escolarización en general, con suposiciones fundamentalmente desafiantes sobre la 
creación y producción de conocimientos que han limitado el alcance de los conocimientos que se producen y reproducen en las escuelas y comunidades.

En tal sentido, este estudio comprueba que al aplicar diversas estrategias de enseñanza basadas en la didáctica pedagógica de interés del educando y de modos activo participativo, despertará el interés del estudiante por preservar su cultura.

\section{Conclusiones}

La revitalización encuentra su potencial en la educación, por ello para recuperar la identidad cultural y revitalizar las lenguas, se debe promover la inclusión de programas y planes enfocados hacia esta temática. Por ende, la educación intercultural bilingüe viene a ser una fortaleza directa para un proceso exitoso de revitalización, esto implica cambios en la política educativa y en los aspectos paradigmáticos de los profesores en la enseñanza.

Cualquier actividad de los docentes, que conlleve a revitalizar la lengua originaria, sobre todo en la Universidad Nacional Intercultural de la Amazonia, será de gran utilidad para los fines académicos de la Universidad. El contexto mismo, ilustra que para tener egresados competitivos en interculturalidad es necesario prepararlos en la Universidad, por ello, la convivencia de profesores y estudiantes, personal administrativo, autoridades y personal jerárquico de mando medio, deben elevar el estandarte de la educación bilingüe.

Asimismo, los egresados están en la obligación de representar dignamente a la casa superior de estudios que los formó, desde el enfoque intercultural. El egresado de la Carrera de Educación, tendrá los recursos suficientes para enseñar a estudiantes de educación básica regular en valores culturales, etnolingüísticas e identidad de sus pueblos.

\section{Referencias Bibliográficas}

Ávila, A. (2018). Lenguas indígenas y enseñanza de las matemáticas: la importancia de armonizar los términos. Revista Colombiana de Educación, (74), 177-195.

Declaración Universal de Derechos Humanos. Aprobada por la res. 217 A (III) de la Asamblea General de las Naciones Unidas, Paris, 10 de diciembre de 1948.

Embriz, O., A. y Zamora, A., O. (2012) (Coor.) Lenguas Indigenas Originarias en Riesgo de Desaparición. México: Instituto Nacional de Lenguas Indígenas.

Estatuto de la Universidad Nacional Intercultural de la Amazonía. Resolución 244-2016UNIA-CO. Yarinacocha, Perú, 19 de agosto de 2016.

Fitzgerald, C. Understanding Language Vitality and Reclamation as Resilience: A Framework for Language Endangerment and "Loss" (Commentary on Mufwene). Language, 93(4): e280-297. DOI: 10.1353/lan.2017.0072.

Hermes, M. Bang, M. and Marin A. (2012). Designing Indigenous Language Revitalization. Harvard Educational Review. 82. 3.

Hernández, S., R., Fernández-Collado, C. \& Baptista, L., P. (2006). Metodología de la Investigación. 4 ed. Ciudad de México, McGraw-Hill. 
Herrán, G., A. y Rodríguez, B., Y. (2017). Indicadores de supervivencia y muerte de culturas y lenguas indígenas originarias en contextos hispanohablantes excluyentes: la enseñanza como clave. Revista Ibero-americana de Educación. 73, núm. 1, pp. 163184.

Jiménez, A., D. (2017). De la voz a la escritura: (auto) traducción y (re)creación en la poesía bilingüe (chaima-español) de Domingo Rogelio León. Mutatis Mutandis. 10, No.1. 2017, 250-270. Doi 10.17533/udea.mut.v10n1a11.

Kroskrity, P. (2018). On recognizing persistence in the Indigenous language ideologies of multilingualism in two Native American Communities. Language \& Communication 62, B, 133-144. Doi: https://doi.org/10.1016/j.langcom.2018.04.012.

Ley $\mathrm{n}^{\circ} 27250$ Ley que crea la Universidad Nacional Intercultural de la Amazonía, Lima., 09 de diciembre de 1999.

Ley $\mathrm{N}^{\circ} 29735$ Ley que regula el uso, preservación, desarrollo, recuperación, fomento y difusión de las lenguas originarias del Perú. Perú.22 de julio de 2016.

MINEDU. (2017) Documento original de lenguas originarias del Perú. Disponible en: http://www2.minedu.gob.pe/filesogecop/DNL-version\%20final\%20WEB.pdf

Ramírez, V., B. y Suárez, V., P. (2016). Conocimiento y aprendizaje de lenguas indígenas en bachilleratos rurales y urbano del estado de puebla, México. Ra Ximhai, 12, 6. 377387.

Rukmini Becerra-Lubies, Simona Mayo \& Aliza Fones (2019): Revitalization of indigenous languages and cultures: critical review of preschool bilingual educational policies in Chile (2007-2016), International Journal of Bilingual Education and Bilingualism, DOI: $10.1080 / 13670050.2018 .1563584$

Santacana, M., J. (2014). Patrimonio, Educación e Historia: el poder invisible del pasado. En Prats, J., Barca, I., \& López, F., R. (Eds.). Historia e identidades culturales. Portugal: CIED, Universidade do Minho.

Stanton, C., Hall, B. and Carjuzaa, J. (2019). The Digital Storywork Partnership: Communitycentered social studies to revitalize Indigenous histories and cultural knowledges. The Journal of Social Studies Research. 43, 2, 97-108. Doi: 10.1016/j.jssr.2018.08.001

Turra, O. y Ferrada, D. (2016). Formación del profesorado en la lengua y cultura indígena: una histórica demanda educativa en contexto Mapuche. Educ. Pesqui.42, 1, 229242. doi.org/10.1590/S1517-9702201603141205.

UNESCO. (2003). Vitalidad y peligro de desaparición de las lenguas. Reunión Internacional de Expertos sobre el programa de la UNESCO "Salvaguardia de las Lenguas en Peligro.

Uriarte, J. (2006). Construir la resilencia en la escuela. Revista de Psicodidactica. 11- 1. 
Anexos

Tabla 1. Estadístico de la prueba de Revitalización de Lenguas Indígenas

\begin{tabular}{lcccccccccc}
\hline & \multicolumn{3}{l}{ Prueba de Entrada - Pre test } & \multicolumn{3}{c}{ Prueba de Salida - Pos test } \\
\hline Media & Desviación & Moda & Máximo & Mínimo & Media & Desviación & Moda & Máximo & Mínimo \\
& & & & & & & & & & \\
\hline $\mathbf{9 . 7 3}$ & 14.14 & 12 & 14 & 3 & 16.6 & 1.76 & 17 & 20 & 13 \\
\hline
\end{tabular}

Tabla 2. Estadísticos de Prueba de Revitalización de Lenguas Originarias

\begin{tabular}{lccccc}
\hline \multicolumn{2}{c}{$\begin{array}{c}\text { Prueba de Entrada de Revitalización de Lenguas } \\
\text { Indígenas }\end{array}$} & \multicolumn{2}{c}{$\begin{array}{c}\text { Prueba de Salida de } \\
\text { Revitalización de Lenguas } \\
\text { Indígenas }\end{array}$} \\
\hline Nivel & Rango & Frecuencia & Porcentaje & Frecuencia & Porcentaje \\
\hline Inicio & $(1-10)$ & 10 & $45 \%$ & 0 & $0 \%$ \\
\hline Proceso & $(11-12)$ & 12 & $55 \%$ & 3 & $14 \%$ \\
\hline Logro previsto & $(13-16)$ & 0 & $0 \%$ & 13 & $27 \%$ \\
\hline Logro & & & & & \\
destacado & $(17-20)$ & 0 & $0 \%$ & 6 & $\mathbf{1 0 0}$ \\
\hline & & & & $\mathbf{2 2}$ \\
\hline
\end{tabular}

